\title{
La Modernidad democrática como religión: una lectura intertextual de la crítica de Gómez Dávila en Textos
}

\section{[Democratic Modernity as a Religion: An Intertextual Interpretation of Gómez Dávila's Critique in Textos]}

\author{
TomÁs FELIPE MolinA \\ Universidad de Granada \\ tomas.molina123@gmail.com
}

\begin{abstract}
Resumen: Nicolás Gómez Dávila caracteriza la Modernidad democrática como una época gnóstica. En este artículo pretendo reconstruir esta caracterización a partir de una lectura intertextual de Textos. Desde una filosofía de la historia ( $9^{\circ}$ texto) y desde una antropología filosófica ( $5^{\circ}$ texto) Gómez Dávila interpreta fenómenos históricos como la Modernidad y la democracia ( $6^{\circ}$ texto) con una perspectiva que privilegia lo religioso. Su conclusión es que en la Modernidad democrática el ser humano se ha arrogado atributos divinos, es decir, se ha divinizando. Así, intentaré mostrar cómo funciona la intertextualidad de Textos en la crítica a la Modernidad democrática y ofreceré además algunas consideraciones críticas.
\end{abstract}

Palabras clave: divinización, teología, política, gnosticismo, soberanía

\begin{abstract}
Nicolás Gómez Dávila characterizes democratic Modernity as a gnostic age. This article pretends to reconstruct his characterization by way of an intertextual reading of Textos. Gómez Dávila's thesis is derived from a philosophy of history and a philosophical anthropology; both allow him to interpret Modernity from a religious perspective. His conclusion is that democratic Modernity bestows human beings unlimited sovereignty, which for him means gnostic divinization. Thus, I intend to explain how intertextuality works in the assessment of democratic Modernity and put forward a few critical considerations.
\end{abstract}

Key words: divinization, theology, politics, Gnosticism, sovereignty

\section{Introducción}

Nicolás Gómez Dávila (1913-1994) es uno de los pensadores y estilistas más importantes en la historia de Colombia. Autores tan variados como Abad Torres 2008, Severino 2001, Volpi 2005 y Reinhart Maurer (Pizano 1991) han reconocido el valor de su obra, la cual puede leerse como un intento de comprensión y crítica de la Modernidad democrática con todos los fenómenos que la acompañan y le dan vida, como la Ilustración, la ciencia, la técnica moderna y la secularización. En esto no es original. La Modernidad ha tenido críticos desde sus orígenes: católicos, escépticos, románticos o historicistas han desarrollado a lo largo de siglos críticas bien conocidas de ella. Tal vez la contribución de 
Gómez Dávila consista justo en condensar toda esta tradición crítica de una forma estilísticamente brillante y filosóficamente intensa. El colombiano es, en efecto, un católico escéptico, romántico e historicista que duda de manera radical —es decir, de la raíz - de la Modernidad democrática. Su tesis central, como se verá aquí, es que la esencia de dicha época es la divinización del ser humano.

Este artículo tiene un propósito explicativo y crítico. En primer lugar, busco reconstruir el pensamiento de Gómez Dávila para mostrar las bases hermenéuticas de su crítica a la Modernidad democrática. Esto quiere decir que me enfocaré sobre todo en Textos, pues de allí mana el grueso de dichas bases. Acercarse a ese libro no es fácil porque no tiene una estructura obvia ni ofrece pistas sobre qué estrategias de lectura podemos seguir para comprenderlo. Por lo tanto, propongo al lector una hipótesis de lectura que busca iluminar la inicialmente invisible relación entre los textos de Textos: se conectan entre sí intertextualmente; de hecho, los unos permiten una lectura más exacta de los otros. Lo anterior significa que los textos no se deben leer en forma individual como si estuvieran cerrados herméticamente, sino como parte de un universo discursivo que se desarrolla en otros textos. Así pues, en este artículo pretendo mostrar que el texto que trata sobre la filosofía de la historia (texto $9^{\circ}$ ) nos muestra el esquema interpretativo que Gómez Dávila utiliza para entender hechos históricos y políticos como la democracia, el ser humano y la Modernidad. En este caso, se echa mano de la Iglesia como esquema hermenéutico. Luego probaré que, a partir de lo anterior, Gómez Dávila desarrolla su idea del homo religiosus (texto $5^{\circ}$ ) como base antropológica para interpretar al ser humano. Por último, se intentará señalar que ambos elementos - filosofía de la historia y homo religiosus - son la clave hermenéutica que le permite al colombiano entender la democracia (texto $6^{\circ}$ ) como religión gnóstica. ${ }^{1}$

En las conclusiones ofrezco además algunas consideraciones críticas. Si la tesis de Gómez Dávila es cierta, entonces la Modernidad democrática se basa en la idea de una soberanía humana total, es decir, que no hay autoridad alguna por encima de las personas. Eso implicaría,

\footnotetext{
${ }^{1}$ En Textos, Gómez Dávila no señala propiamente a la Modernidad como una época gnóstica. Eso lo hará en los Escolios. Sin embargo, sí habla de una "época democrática" en la que el comunismo, el capitalismo y el Estado soberano son los fenómenos centrales. Así, no es descabellado señalar que dicha época es la Modernidad misma. Por lo demás, el argumento que se utiliza en Textos contra la democracia es esencialmente el mismo que se desarrolla en los Escolios contra la Modernidad, pues allí caracteriza a dicha época como gnóstica. Esto se mostrará en las conclusiones del artículo.
} 
como se verá más adelante con detalle, que, según el colombiano, el ser humano se ha arrogado características divinas y que Dios ha muerto. En otras palabras, la persona ha asumido el papel que Dios tenía en la Edad Media: ser el soberano del universo, el fundamentador del ente y de los valores, etc. Empero, la Modernidad necesita aún de alguien que desempeñe el papel de Dios aparte del individuo, por lo que su muerte no ha significado el inicio de una soberanía humana ilimitada. Al contrario: el ser humano moderno ha creado una serie de sustitutos de Dios que limitan su propia soberanía.

Antes de continuar es preciso hacer una aclaración. Abad Torres 2010, Serrano Marín 2015 y Rabier 2016 han realizado un trabajo valiosísimo sobre el problema del gnosticismo en Gómez Dávila. Se han encargado de explicar la relación histórica y filosófica de la crítica de este autor con el gnosticismo. Todavía hoy, los suyos son algunos de los pocos trabajos que se encuentran sobre este tema. Mi artículo, aunque sin duda se inspira en esas fuentes, propone un aporte académico nuevo: además de mostrar la crítica del colombiano, también muestra en forma breve cómo el autor alcanza su crítica de la Modernidad democrática por medio de una filosofía de la historia y de una antropología religiosa. En otras palabras, mi contribución es la de mostrar una interpretación intertextual. Esto es algo que sólo Mosebach 2017 ha sugerido, aunque sin desarrollarlo.

\section{Reconstrucción de la hermenéutica de Gómez Dávila \\ 1.1. Acercarse a Textos}

Para explicar el enfoque intertextual de este trabajo vale la pena hacer primero unas aclaraciones sobre el libro que analizaré en esta sección: Textos. Si nos guiamos por su título, como señala Torres Duque 1995, podríamos concluir que puede contener cualquier cosa. En su interior, los diferentes textos sólo se separan por hojas en blanco: no hay títulos, subtítulos, ni guías implícitas sobre cómo leerlo. Apenas hay un epígrafe y un breve proemio en uno de los textos, de manera que la contextualización inicial resulta difícil en extremo. Consta de diez partes, si se pueden llamar así, que tratan sobre una o varias materias: antropología filosófica, la libertad humana, la relación entre el ser humano y Dios, la muerte, los valores, la filosofía de la historia, la democracia y la conciencia humana.

El estilo del libro podría caracterizarse como paratáctico. Esto quiere decir que las oraciones se conectan más mediante conjunciones coordinadas que por subordinadas, de manera que todas tienen el mismo

Revista de Filosofía Diánoia, vol. 65, no. 84 (mayo-octubre de 2020) e-ISSN: 1870-4913 • DOI: https://doi.org/10.22201/iifs.18704913e.2020.84.1612 
peso. Por ejemplo, en el siguiente pasaje Gómez Dávila casi no subordina ninguna de las oraciones por medio de las conjunciones usuales en español ("por lo tanto", "entonces", "porque", "donde", etc.):

El hombre prueba evidencias de fracaso sin herir su carne intacta. Ni quien yerra, ni quien se envilece, ni quien peca, mutila instrumentos de victoria. No urge ser, para sobrenadar en la lodosa riada, ni sabio, ni santo, ni noble; y usualmente es menester no serlo. La vida ignora incongruas amenazas. (Gómez Dávila 2002, p. 92)

Este estilo ya aparece en Notas, libro publicado algunos años antes, aunque en Textos se desarrolla en otro nivel estético. Mientras que en el primero el autor todavía habla con una voz más convencional, en el segundo adopta un lenguaje notablemente refinado, metafórico y simbólico, de una deslumbrante precisión y belleza poética. Su efecto estético no puede desligarse del contenido de la obra. En Textos, Gómez Dávila despliega una visión de mundo que se opone de manera radical a la Modernidad. Sin embargo, no pretende criticar los principios de nuestra época con un discurso claro y distinto en el que las ideas y sus conexiones quedan encadenadas en formas obvias, sino por medio de un lenguaje de tendencia poética que en ocasiones se asemeja más a un himno simbolista que a un discurso filosófico convencional. Porque no hay subordinación, tampoco hay argumentación en el sentido usual de la Ilustración. Las premisas no desencadenan una serie de conclusiones por medio de conjunciones del tipo "aquí se dice esto, luego podemos decir aquello", sino que se presentan más bien varias afirmaciones cuya conexión es, hasta cierto punto, misteriosa, precisamente por la ausencia de subordinación. Por lo tanto, el estilo del libro de algún modo se desliga de la Modernidad.

El intérprete se topa aquí con una doble dificultad: por un lado, no tiene claro cómo entender la estructura de Textos puesto que, como señalé, no hay ninguna guía obvia que permita darle sentido al conjunto de los diferentes textos que componen el libro; por otro lado, el estilo del libro es muy refinado y hasta cierto punto misterioso, de manera que comprender su lenguaje demanda una lectura extremadamente lenta y detallada. Veamos el primer problema. En el libro, los temas y textos se suceden los unos a los otros sin conexiones obvias entre sí. Eso es justo lo que sucede también con las oraciones en el estilo paratáctico del autor: no hay una conexión obvia entre unas y otras, aunque, de hecho, la hay: de otra manera los párrafos no tendrían una lógica interna. Lo mismo pasa con el libro: presenta un alto 
grado de intertextualidad que le otorga una lógica interna, aunque ésta no resulte clara al principio. Aquí quiero mostrar que Gómez Dávila propone una interpretación religiosa de la democracia (texto $6^{\circ}$ ) porque él mismo interpreta al ser humano (texto $5^{\circ}$ ) y a la historia desde una perspectiva religiosa (texto $9^{\circ}$ ).

Con el término "intertextualidad" aquí se quiere decir que los fragmentos y textos de Gómez Dávila no son autónomos, sino que se ubican en un universo literario con el cual están en relación dialógica. En otras palabras, los textos del filósofo colombiano tienen una conexión con otros textos. Ésta no es una suposición arbitraria. En primer lugar, el título de la obra más conocida de Gómez Dávila ya la sugiere: sin duda, Escolios a un texto implícito apunta a que las ideas que allí se encuentran se refieren siempre a una fantasmal obra implícita con la que permanecen en constante diálogo. En segundo lugar, en Textos el autor señala que su estudio sobre la democracia es un "centón", es decir, un trabajo hecho a partir de escritos de una tradición. Por ello, en sus palabras hay inevitablemente palabras de otros, esto es, intertextos. En este artículo sugiero que dichos intertextos son también otros textos del mismo libro. En tercer lugar, el epígrafe inicial de Notas, el primer libro que publicó Gómez Dávila, nos dice que sólo podemos comprender su contenido si nos dedicamos a indagar sobre lo que dice, es decir, si nos referimos a los textos que dichas notas comentan, esto es, si leemos textos de otros. Por lo tanto, en las tres obras principales de Gómez Dávila hay una intertextualidad clara a la que el autor se refiere. Uno podría decir que las pruebas anteriores apuntan a que el autor sugería que se hiciera una lectura intertextual de su obra.

Hay tres maneras de entender la intertextualidad del filósofo colombiano. La relación con textos ajenos es la primera y más obvia: casi todos los comentaristas de la obra la han advertido de manera intuitiva. Su corolario es que, para entender al filósofo, es preciso ir a las fuentes de las que su pensamiento bebe y que permanecen relativamente ocultas. Sin embargo, este primer tipo de intertextualidad — que podríamos caracterizar como la relación entre los fragmentos y un hors-texte- tendrá aquí un papel secundario. En Rabier 2014a y Rabier 2014b se explora el asunto de manera profunda y no es mi propósito ahondar en él. ${ }^{2}$ Esto se debe a que mi artículo se propone hacer énfasis en el segundo tipo de intertextualidad, es decir, en la relación que hay entre los fragmentos o secciones de una misma obra del colombiano. Así, quiero mostrar que en Textos encontramos hilos conductores que unen a los textos

\footnotetext{
${ }^{2}$ Véanse también Molina 2019a y Molina 2019b.
} 
de la obra con otros textos de la misma obra. Esto implica que una interpretación intertextual debe dar con una narrativa más completa que aquella que surge de una lectura aislada de los textos del colombiano. Este trabajo pretende mostrar, mediante la lógica intertextual, cómo el autor llega a interpretar la democracia como un fenómeno religioso.

El tercer tipo de intertextualidad es el que se desarrolla entre las distintas obras de Gómez Dávila. Esto quiere decir que hay una relación entre Escolios y Textos. Ya Francisco de Brigard examinó esta idea cuando sostuvo que, según el mismo autor, "el texto implícito está contenido en las páginas 61 a 100 de su libro Textos I y su tema es la democracia" (Pizano 1991, p. 12). Dicho de otro modo, el texto implícito de los Escolios está en Textos. Esta intertextualidad también tiene un carácter secundario en este artículo. Sólo en las páginas finales aludiré a las conexiones que hay entre las ideas desarrolladas en Textos y Escolios.

\section{2. La Iglesia católica y la antropología religiosa como bases hermenéuticas de la crítica a la Modernidad}

\subsection{La Iglesia católica como esquema de comprensión histórico}

En el noveno texto de Textos, Gómez Dávila afirma que nuestra comprensión del mundo está determinada históricamente. No es posible escapar de la historia para alcanzar principios puros. En efecto, para nuestro autor el pasado mismo nos determina de manera ineludible y es responsable de la manera en que percibimos el mundo. Nuestra conciencia se enmarca en una estructura histórica y es producto de la historia. Incluso lo que sentimos - o al menos cómo lo sentimos- nos viene del pasado. Así, puede que todos los seres humanos sean capaces del amor o de la envidia, pero las maneras en los que los sentimos y el porqué los sentimos "son el fruto de elaboraciones colectivas" (Gómez Dávila 2002, p. 116).

Sin embargo, Gómez Dávila cree que nuestra razón no se resigna a la condición histórica del ser humano y, por eso, "se insubordina contra la opresión de decisiones vetustas" (Gómez Dávila 2002, p. 117). Aunque la razón pura intente desligarse de su condición concreta para observar la historia desde un peñasco inmóvil, desde una perspectiva privilegiada y ahistórica, se trata de una hazaña imposible: toda teoría está atada a la historia. "El artificio más abstracto radica en la impura confusión de la historia" (Gómez Dávila 2002, p. 117). Al intentar alcanzar una posición objetiva y ahistórica, simplemente proyectamos nuestra situación actual en el pasado. Debemos ser conscientes de nuestra condición histórica para tratar de usarla de manera inteligente. En otras 
palabras, cuando negamos o ignoramos la historicidad del ser humano quedamos atrapados en los tópicos del día, pero cuando la pensamos en forma inteligente podemos escapar parcialmente de ellos. El historiador puede hacer esto si logra integrar nuestra vida en un contexto más ancho que el de los tópicos del día (Gómez Dávila 2002, p. 119).

¿Cómo puede alcanzar el historiador el propósito anterior? No basta que haga un catálogo minucioso de hechos ni que nos narre con elocuencia lo sucedido en el pasado (Gómez Dávila 2002, p. 118), pues el historiador no escapa automáticamente, por ser historiador, de su condición inmediata para integrar su vida y la nuestra en el universo de la experiencia humana, ya que en principio "añade a las limitaciones de su carne las limitaciones de su tiempo" (Gómez Dávila 2002, p. 118). En efecto, la posición por defecto de todo historiador es la de imponer las perspectivas de su época y de su persona a la comprensión del pasado, puesto que naturalmente lo pensamos desde nuestra posición histórica concreta. El historiador necesita entonces una herramienta que le permita pensar más allá de su instante para que pueda ver el pasado como el pasado se vio a sí mismo. Esa herramienta es, para Gómez Dávila, un esquema filosófico.

El esquema tiene que respetar la historicidad de los hechos. Ésta consiste en que cada hecho sucedió en un contexto particular, en una condición concreta: no existe en el vacío; el hecho histórico es tautológicamente histórico, esto es, pertenece a un contexto histórico particular, en un lugar específico del tiempo. Para comprenderlo tal y como fue no basta con observarlo desde nuestra condición concreta (nuestro contexto), pues sólo se la impondremos para ignorar el contexto particular del hecho. En cambio, el esquema filosófico permite observar correctamente esa historicidad, de una manera en que no falsificamos el pasado. Esto quiere decir que el esquema propone una especie de punto de observación privilegiado en el que la historicidad de los hechos es comprensible desde su propio contexto. El esquema pretende reemplazar nuestra posición natural de conciencias individuales en una situación histórica por una en la que podamos incorporar los datos presentados a conciencias pasadas como si fuésemos esas conciencias pasadas. El esquema filosófico nos conecta con otras conciencias, con otros mundos. Es un puente que nos permite adentrarnos en la historia, para comprenderla sin imponerle nuestros prejuicios contemporáneos. Esto se puede entender mediante una metáfora que usa el filósofo colombiano: el diseñador del esquema funciona como un cartógrafo que aúna itinerarios de viajeros desacordes con la mínima distorsión posible (Gómez Dávila 2002, p. 119). 
Gómez Dávila nos dice que, aunque en la práctica existen tantos esquemas como filosofías de la historia, en realidad éstas suelen ser maneras distintas de emplear un mismo esquema o de combinarlos. Si intentamos buscar la esencia básica de los esquemas existentes, nos quedan apenas tres. El primero y más antiguo es el esquema del providencialismo histórico, el cual consiste en atribuir a "una divina providencia, como a su causa inmediata y constante, la totalidad de la historia" (Gómez Dávila 2002, p. 121). Contrario a lo que uno podría esperar de un homo religiosus como el colombiano, este esquema le resulta poco convincente. Su utilidad explicativa es nula justo porque no puede explicar nada. La lógica del providencialismo es circular: el hecho acontece porque la providencia lo decide; y la prueba de que la providencia lo ha decidido es que el hecho acontece. Este esquema puede intentar justificar ciertos hechos atribuyéndoles como causa la voluntad divina pero, aparte del uso político y psicológico que pueda tener, en materia de hermenéutica histórica su utilidad es nula. Gómez Dávila es claro: "explicar por medio de la divina providencia es, estrictamente, no haber dicho nada" (Gómez Dávila 2002, p. 122).

El segundo esquema es el progresista. Aquí se reemplaza el plan providencial por una finalidad interna (Gómez Dávila 2002, p. 122). Su lógica es la siguiente: como la historia tiene un propósito final, es preciso ordenar los hechos de manera que podamos entender cómo es que la historia se ha dirigido hacia su propósito. En palabras del autor, aquí cada hecho se trata "como mero eslabón de una cadena de causas que convergen hacia el efecto elegido" (Gómez Dávila 2002, p. 122). El hecho se subordina a la finalidad. Esta finalidad es el progreso en cualquiera de sus formas: la democracia liberal, el comunismo, la nación, la humanidad, etc. Para Gómez Dávila, este esquema distorsiona necesariamente la historia, porque para ordenar todo hecho hacia un propósito rígido es preciso tomar del hecho sólo lo que en efecto podría considerarse que conduce a la finalidad que se le atribuye a la historia. Lo demás se omite y, por lo tanto, carece de explicación e incluso de realidad, al menos según la lógica del esquema. Sin embargo, hay algo igual de malo aquí: le niega al hecho histórico su propia finalidad. Ésta es en lo básico una negación de la historicidad de los hechos, tal y como Gómez Dávila la entiende: que el hecho pertenece a un contexto concreto y debe entenderse justo prestándole atención en vez de someterlo a un propósito arbitrario y exterior, como propone el esquema progresista.

El tercer esquema es el reduccionista, que propone la reducción de los hechos a un solo factor o a un grupo de factores; por ejemplo, el instinto sexual, la constitución étnica o un comportamiento social (Gómez 
Dávila 2002, p. 122). Para que este esquema tenga sentido, es preciso que el factor sea universal: de otro modo no es posible aplicarlo a la historia en su totalidad. Tienen que compartirlo los romanos, los chinos, los franceses contemporáneos, los colombianos; en fin, tiene que pertenecer a la especie entera. Además, todos los demás factores deben someterse al factor determinante, es decir, deben ser funciones de él. De otro modo, habría un universo heterogéneo de factores explicativos en vez de la determinación de uno solo. El problema de este esquema es que, aunque explicar un hecho mediante un factor como los mencionados puede ser factible en ciertos contextos, someter todo hecho a un solo factor resulta abusivo en términos hermenéuticos. Además, crea una ilusión de ahistoricidad: la historia queda explicada por un factor exterior al tiempo, independiente del contexto. En realidad, aquí también se niega la historicidad del hecho, pues todo contexto se somete en forma arbitraria al factor predominante. Así, la historia entendida desde la historicidad del hecho mismo desaparece para que sólo quede una ley abstracta que la rige.

Después de explicar por qué las combinaciones de los esquemas no pueden resolver el problema, Gómez Dávila vuelve a afirmar que, como somos seres históricos, ninguno de los esquemas puede ser ahistórico. De hecho, "la obra histórica acostumbra ser más fiel testimonio sobre el tiempo en el cual se escribe, que sobre el tiempo sobre el cual fue escrita" (Gómez Dávila 2002, p. 125). No se puede explicar un hecho histórico sin anacronismos, porque todo lo explicamos a partir de lo que somos. "Lo totalmente extraño es infranqueable a nuestra comprensión humana" (Gómez Dávila 2002, p. 127). ¿Cómo podemos entonces entender un hecho histórico como la Modernidad? Gómez Dávila propone al menos dos requisitos. Si la comprensión histórica tiene como condición poner al sujeto y al objeto en situaciones idénticas (de otro modo no logramos comprender el pasado en su situación concreta), tiene que ser producto de una larga tradición que logre conectar el pasado con el presente. Pero esa misma tradición tiene que ser una realidad objetivada en la que participemos; no puede ser sólo una construcción imaginada y ajena. Quien quiere comprender debe pertenecer a y heredar la tradición que le permita comprender. Sin embargo, esa herencia no es privilegio de una élite, puesto que eso limitaría lo que la tradición puede recoger y disminuiría su capacidad como aparato de comprensión histórica. La tradición tiene que ser una colectividad abierta que hereda sus evidencias a quien esté dispuesto a recibirlas (Gómez Dávila 2002, p. 130). La tradición, en fin, es la prolongación de experiencias pasadas en la situación concreta de cada individuo.

Revista de Filosofía Diánoia, vol. 65, no. 84 (mayo-octubre de 2020) e-ISSN: 1870-4913 • DOI: https://doi.org/10.22201/iifs.18704913e.2020.84.1612 
Para Gómez Dávila, la Iglesia católica es lo más cercano a su propuesta: es una larga tradición que reúne incluso las tradiciones de sus enemigos y que, al mismo tiempo, se plasma en una realidad objetivada de la que podemos participar. Está abierta a todos. De hecho, para el bogotano en la Iglesia perdura incluso "la postración del primer simio ante la impasibilidad de los astros" (Gómez Dávila 2002, p. 135), de manera que es la tradición que mejor reúne la experiencia religiosa, política y social del ser humano. Si es así, nos puede colocar en una situación similar a la del hecho histórico que anhelamos comprender, de manera que no le impongamos nuestras ideas, nuestra condición histórica particular. Pero ¿qué es lo que extrae Gómez Dávila de la Iglesia como herramienta hermenéutica para la comprensión de la historia?

\subsubsection{El ser humano como ser religioso}

Vamos al quinto texto de Textos. Allí Gómez Dávila desarrolla una antropología a partir de su hermenéutica católica. Lo que quiere es hacer énfasis en la esencia religiosa del ser humano. Su tesis principal es que el humano se distingue de los animales no por su inteligencia ni por su habilidad para someter al mundo, pues ésa es una diferencia de grado que no alteraría la continuidad biológica entre nosotros y ellos. Aquí hay un abismo ontológico. Lo que en verdad explica la singularidad del humano es su contacto con lo divino: cuando el individuo siente a Dios, su humanidad ha nacido. Dios no se entiende aquí en un sentido circunscrito por la doctrina católica ortodoxa, sino en el sentido más amplio posible de la Iglesia entendida como tradición, esto es, como recolectora de todas las religiones: Dios es una realidad del orden sobrenatural que se revela como horror, majestad, esplendor y peligro al primer simio que se postra y se convierte en humano. En las poéticas palabras del colombiano:

En el silencio de los bosques, en el murmullo de una fuente, en la erguida soledad de un árbol, en la extravagancia de un peñasco, el hombre descubre la presencia de una interrogación que lo confunde.

Dios nace en el misterio de las cosas.

Esa percepción de lo sagrado, que despierta terror, veneración, amor, es el acto que crea al hombre, es el acto en que la razón germina, el acto en que el alma se afirma.

El hombre aparece cuando Dios nace, en el momento en que nace y porque Dios ha nacido. (Gómez Dávila 2002, p. 48)

Esa última oración es la más importante: el ser humano sólo puede ser humano por Dios mismo, porque Dios existe. Para decirlo de manera 
concreta: la esencia del humano es el contacto con lo divino. Como apunta Mircea Eliade al explicar esta clase de hermenéuticas religiosas tradicionales: "la vida no es posible sin una apertura hacia lo trascendente; en otras palabras, los seres humanos no pueden vivir en el caos" (Eliade 2015 p. 34). Una vez que hay contacto con lo divino, el ser humano puede ordenar su vida, puede orientarse. Como dice también Eliade: "la revelación de un espacio sagrado hace posible la obtención de un punto fijo y, por tanto, la orientación en el caos de la homogeneidad; permite 'fundar el mundo' y vivir de un modo real" (Eliade 2015 p. 23). Así, lo divino no es lo totalmente Otro, lo infinitamente inaccesible, sino lo Otro que permite iluminar, crear y dar sentido al mundo del ser humano. Sólo por medio de nuestro contacto con Dios tenemos un mundo donde morar, en el sentido de un universo ordenado y con valor. La mundanidad de las personas tiene como condición de posibilidad la existencia de Dios.

Todo lo anterior surge de la Iglesia como esquema de comprensión histórico. En efecto, Gómez Dávila no propone la Iglesia como un mero ejercicio intelectual. Al contrario, es parte fundamental de su comprensión. De ahí que Martin Mosebach haya señalado que, pese a su pasión por la historia, el colombiano buscaba un resquicio que le permitiera escaparse de las limitaciones de su propio tiempo y de su lugar geográfico, de manera que pudiese formular una antropología eterna contra la Modernidad (Mosebach 2017). Ese resquicio era justo la filosofía de la historia que ya se explicó: la Iglesia como esquema de comprensión le permite a Gómez Dávila definir al humano como un ser principalmente religioso.

Para la Iglesia, lo característico del humano es su contacto con Dios. Por lo tanto, toda explicación de lo que el ser humano es tiene que privilegiar su relación con lo divino.

\subsubsection{Hermenéutica religiosa para interpretar la democracia}

Aquí nos conectamos con el sexto texto de Textos, es decir, el de la crítica a la Modernidad democrática. Gómez Dávila nos dice allí que las filosofías de la historia se proponen definir la relación entre el individuo y sus actos, y la manera en que la filosofía hace eso determina su explicación del universo (Gómez Dávila, 2002 p. 59). Por "acto" se refiere a lo que entendemos en el lenguaje cotidiano (la acción de hacer), así como a los objetos de la conciencia y la definición de su estatus metafísico. Por medio de la tradición de la Iglesia, la filosofía de la historia de Gómez Dávila se propone definir la relación entre el ser humano y sus actos. Esto tiene como resultado que el escogimiento de tal o cual 
valor o el desarrollo de tal o cual principio social resultan mediados por una opción religiosa. Esto es también el resultado de la antropología de nuestro autor. Al definir al humano como ser religioso, resulta natural señalar que la relación entre el ser humano y sus actos está mediada por una noción de Dios, es decir, por una opción religiosa.

En estas páginas, Gómez Dávila repite el argumento que ya ofreció en su texto sobre la filosofía de la historia: las filosofías de la historia suelen sacrificar la trama para ordenar los hechos de un modo arbitrario que falsea al hecho histórico mismo. Se necesita una filosofía de la historia que ordene las configuraciones de motivos mediante un principio general que no las falsifique, es decir, que respete su historicidad. El que Gómez Dávila elige es el de la opción religiosa, pues la Iglesia privilegia el carácter religioso del ser humano. Empero, es posible pensar que al final este esquema no resulta ser más que uno reduccionista en el que todo se somete a un solo factor histórico que ordena los hechos de manera arbitraria y en el que todos los demás factores son simples funciones del factor predilecto, esto es, la opción religiosa. Sin embargo, lo que se propone es más ingenioso: la comprensión histórica necesita establecer que cada hecho es fin de sí mismo, pero al mismo tiempo tiene que ordenar los hechos para mostrar qué propósito más allá de sí mismos están cumpliendo (Gómez Dávila 2002, p. 128). Nos dice, además, que sólo la Iglesia cumple esa condición de la comprensión. A diferencia del esquema reduccionista en el que el hecho individual nunca es fin de sí mismo y, por lo tanto, la causa y el propósito de todos los hechos se reducen a uno solo, en el esquema de Gómez Dávila los hechos sí pueden ser fin de sí mismos, pero están orientados por una opción religiosa previa. Es decir, hay un reconocimiento de la historicidad del hecho y, al mismo tiempo, el sometimiento a un principio general que lo ordena y lo explica.

Una objeción obvia que se puede presentar a esto es que, aunque el esquema de la Iglesia pretende mostrar los hechos en su condición particular, hay condiciones concretas en las que los individuos no tienen una motivación religiosa. Por eso no podría decirse que sus actos son siempre una función de su ámbito religioso. En este sentido, Gómez Dávila estaría imponiéndoles una motivación que no tienen. El colombiano sólo puede escapar de esto señalando que "el individuo ignora usualmente la opción primigenia que lo determina" (Gómez Dávila 2002, p. 61). Esa apelación al inconsciente tal vez no sea válida, pero es necesaria para conservar la integridad de su esquema; es la única manera de sostener en forma coherente que "Todo acto se inscribe en una multitud simultánea de contextos; pero un contexto unívoco, in- 
moto y último, los circunscribe a todos. Una noción de Dios, explícita o tácita, es el contexto final que los ordena" (Gómez Dávila 2002, p. 61).

$\mathrm{El}$ autor reconoce aquí que, como dice en otro lugar de Textos, los actos del ser humano "nunca son la limpia realización de un valor único. Un rito religioso cumple exigencias estéticas, un proceso económico vela un comportamiento ético, una naturaleza muerta exhibe un programa político" (Gómez Dávila 2002, p. 107). Por ello, las motivaciones de cada acto pueden ser múltiples y también pueden estar, al mismo tiempo, en una multitud de contextos. Sin embargo, Gómez Dávila propone un contexto que los circunscribe a todos, de manera que queden sometidos a un principio general. El contexto último de todo acto humano sería el religioso, pero eso no elimina los demás contextos, ni siquiera los subordina propiamente: circunscribir es ceñir, poner límites. Lo que quiere decir, entonces, es que la opción religiosa, entendida como una noción de Dios, es aquello que pone límites a los demás contextos, lo que les da forma mediante un ceñimiento.

Lo anterior es una consecuencia necesaria de su hermenéutica católica y de su antropología religiosa. Que la esencia del ser humano es religiosa lo sabe Gómez Dávila porque interpreta el mundo a través de lentes católicos. Además, si lo que nos hace humanos es la religión (entendida como lo relativo a lo divino), si lo que permite que el individuo escape del valor único del mundo animal es la irrupción de lo sagrado, y si, en fin, los valores y el mundo mismo son, en última instancia, el resultado de la hierofanía, resulta natural que la opción religiosa circunscriba el significado de los actos humanos. De ahí que el filósofo diga que la "relación entre el hombre y sus actos es una relación mediatizada. La relación entre el hombre y sus actos es relación entre definiciones de Dios y actos del hombre. El individuo histórico es su opción religiosa" (Gómez Dávila 2002, p. 61).

Es posible reformular esa idea del siguiente modo: no hay acto humano que no esté mediatizado. No hay acto cuyo significado no sea relacional, es decir, todo acto se forma o se funda por medio de una referencia a un Otro divino que le da su significado. El acto humano, por lo tanto, no tiene un significado propio, intrínseco. La misma posición crítica de Gómez Dávila está determinada por este principio: su definición de Dios como ser exterior, superior y creador del ser humano pone a la persona como mera criatura suya. Así, los actos del ser humano tienen significado como criatura; están circunscritos por esta definición, aunque en cada situación concreta respondan a múltiples motivaciones diferentes. Cada acto conserva así su historicidad propia, es decir, su condición histórica específica, a la vez que se somete a un principio ge-

Revista de Filosofía Diánoia, vol. 65, no. 84 (mayo-octubre de 2020) e-ISSN: 1870-4913 • DOI: https://doi.org/10.22201/iifs.18704913e.2020.84.1612 
neral que lo explica. Gómez Dávila mismo se interpreta de esta manera: sus actos, e incluso sus escritos, son los de una criatura.

\section{Crítica a la Modernidad democrática}

A partir de las bases anteriores, Gómez Dávila desarrolla su crítica de nuestra época. Esto quiere decir que, por medio de un énfasis en la religiosidad del ser humano, nos explicará la opción religiosa de la Modernidad democrática. Su argumento consiste en mostrar que la antropología democrática define al ser humano como un dios, aunque de manera velada, e incluso insincera. En otras palabras, la opción religiosa de la democracia es una opción por el ser humano como ser divino. Según nuestro autor, en la democracia el ser humano es el soberano, el creador de los valores, el que define lo bueno y lo malo y su propio redentor. Adopta, pues, la posición que Dios tenía en el universo teológico. Veamos con cuidado el proceso mediante el cual se desarrollan estas ideas.

Según Gómez Dávila, la democracia define al ser humano como voluntad soberana y autónoma (Gómez Dávila 2002, pp. 64-65). Esto se debe a dos razones. La primera es que el principio básico de la democracia es la soberanía popular. La democracia pura es una imposibilidad si no se presupone que el pueblo es soberano, es decir, que la autoridad última reside en él. De otro modo, el pueblo no se daría sus propios gobernantes, sino que otro puede decidir por él cuáles son (esto es, la autoridad última). Ahora bien, el pueblo es un conjunto de seres humanos. Sería una contradicción decir que el pueblo es soberano y que los seres humanos no lo son. El pueblo no puede tener atributos esenciales que el individuo no tenga $-\mathrm{o}$ por lo menos de ese supuesto parte la antropología democrática de Gómez Dávila-. Si el ser humano es soberano, entonces no hay un principio exterior que lo determine y, por lo tanto, su esencia (aquello que hace que sea lo que es) es la voluntad autónoma.

Veamos esto de otro modo. Cuando se dice, de acuerdo con la fórmula de Sieyès, que la voluntad del pueblo es siempre la forma suprema de la ley (Schmitt 1985, p. 48), básicamente se afirma que la voluntad del pueblo es el aspecto esencial y determinante en una democracia; no una razón ni unos principios específicos, sino la voluntad. Esto se debe a lo siguiente: someter la voluntad del pueblo a una serie de principios o razones implica afirmar que esos principios tienen una prelación sobre la voluntad del pueblo, es decir, que ésta no es soberana. En otras palabras, contradice el principio democrático básico de 
que la voluntad del pueblo es la forma suprema de la ley. La voluntad democrática es formalmente irrestricta. Entonces, ¿̇se puede sostener una antropología democrática sin recurrir al principio de la supremacía de la voluntad humana? Según Gómez Dávila, la respuesta es negativa: la democracia necesita presuponerla para elevar la voluntad del pueblo a principio político.

Aquí se introduce la opción religiosa de la democracia. La antropología recién descrita implica una relación antagónica con Dios, pues necesariamente lo excluye. No sólo eso: también lo reemplaza. La razón es la siguiente. El colombiano da por sentado que, si hay algo en la esencia del ser humano que supere su propia voluntad, ese excedente lo sujeta a una instancia externa y, por lo tanto, le quita su autonomía. En consecuencia, la democracia no puede reconocer la supremacía de Dios. El poder político último no puede venir de la divinidad, como en las monarquías medievales, sino de la decisión autónoma de los seres humanos. En la democracia, el sujeto adopta el papel que tenía Dios. Aceptar que Dios es el que da el poder político implica resignarnos a ser sus criaturas, es decir, a no ser autónomos (Gómez Dávila 2002, p. 66). En este caso, la esencia humana no podría ser la voluntad; es decir, su esencia estaría determinada por un ser exterior. Por eso, Gómez Dávila dice en los Escolios que "Dios es el estorbo del hombre moderno" (Gómez Dávila 2005a, p. 310). En efecto, si se piensa desde una perspectiva teológica, Dios estorba a quien pretende ser totalmente autónomo: la existencia de Dios lo sujetaría a una instancia exterior. Nuestra época está circunscrita entonces por una opción religiosa: el rechazo de Dios a favor de un sujeto que toma el papel de la divinidad. El ser humano es ahora la fuente del poder político y de los valores.

La anterior tesis no es en particular polémica dado que algunos movimientos emancipatorios de la Modernidad la han sostenido de un modo $\mathrm{u}$ otro, es decir, han propuesto que sólo mediante el rechazo de Dios el humano será verdaderamente libre y soberano. En la tesis doctoral de Marx podemos encontrar, por ejemplo, que debemos rechazar a los dioses para que la autoconciencia humana sea la deidad suprema (Marx 2019 p. 7). Por eso Bruno Latour señala que desde Hobbes "no es totalmente moderno quien no está de acuerdo con evitar que Dios interfiera con el derecho natural y las leyes de la república. Dios se borra así de la metafísica" (Latour 1991 p. 33). Por lo demás, los demócratas han tenido siempre una relación complicada con Dios justo porque el principio de la democracia es la soberanía popular, no la soberanía de Dios. 
No obstante, es preciso hacer énfasis en que Gómez Dávila no sólo sostiene que hemos excluido a Dios, sino que lo hemos reemplazado. El sujeto democrático moderno es un hombre-dios (Gómez Dávila 2002, p. 62). Ésta es su tesis más difícil de aceptar, porque al menos a primera vista es sin duda concebible una soberanía democrática perfectamente secular. Desde luego, para un lector que partiera de otras premisas hermenéuticas no resulta legítimo deducir que "soberanía" significa lo mismo que "autodivinización". Pero es preciso recordar la lógica con la que aborda Gómez Dávila el problema: su idea es que no es posible escapar de la religión o, más bien, que el ser humano debe interpretarse en términos religiosos. Éste es uno de los principios básicos de la tradición católica. Así pues, la soberanía democrática implica usurpar el lugar de Dios. Para decirlo de manera sintética: Dios era antes la fuente del poder político y de los valores; ahora lo es el ser humano soberano. Por lo tanto, el ser humano tiene el lugar de Dios. Gómez Dávila incluso llega a decir que los individuos democráticos y modernos se arrogan el papel de mesías en la medida en que ellos mismos se encargan de redimirse de su propia miseria por medio del progreso y de la técnica (Gómez Dávila 2002 p. 75).

Si suponemos que la soberanía implica una divinización, ¿por qué hablar de gnosticismo? En primer lugar, porque, como sostiene Abad Torres 2010, "el autor de los Escolios [...] asume el gnosticismo como un fenómeno religioso que no se arrodilla ante Dios, sino ante una nueva divinidad: el hombre". En otras palabras, el gnosticismo implica para Gómez Dávila que el ser humano toma el papel de Dios. Pero hay otra razón: el gnosticismo también ha estado implicado por mucho tiempo en la historia del catolicismo. La tesis de Harnack, como indican Blumenberg y Wallace, es que el catolicismo se construyó en oposición al gran gnóstico Marción, lo que quiere decir que "la formación de la Edad Media sólo se puede entender como un intento de la exclusión del síndrome gnóstico" (Blumenberg y Wallace 1983, p. 130). El gnosticismo sería, en este caso, la contraparte del catolicismo. Si el uno define al humano como un ser cuya esencia es divina (la chispa del Padre que está atrapada en nuestro cuerpo), el otro lo define como un ser cuya esencia es de criatura, es decir, sometida a la voluntad Dios. En el primero, el ser humano es de la misma esencia que la divinidad; en el segundo hay una distancia entre Dios y el humano.

Por lo anterior, Gómez Dávila inicia su texto sobre la democracia con

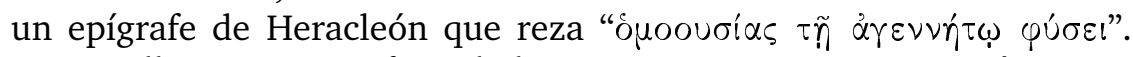
En castellano esto significa "de la misma sustancia que su ingénita naturaleza". Para el pensador bogotano, el sujeto democrático adopta la 
misma posición estructural que Dios tenía en el universo medieval, ${ }^{3}$ es decir, es el amo y señor, la fuente de los valores, el fundamento ontológico de la realidad, la autoridad suprema. Esto sólo puede significar que el humano considera que su sustancia es la misma que la de la divinidad o, para ser más exactos, que el ser humano es Dios. Tal es justo la tesis del gnosticismo, y por eso el sujeto democrático es gnóstico, al menos analógicamente.

\section{Conclusiones}

Hasta aquí hemos visto que Gómez Dávila procede a partir de una filosofía de la historia que privilegia en forma radical —es decir, de raízel factor religioso como elemento explicativo de los hechos históricos. De ahí interpreta al humano como homo religiosus y, por lo tanto, señala que, puesto que lo central en el ser humano es su relación con Dios, todos sus actos deben explicarse sin perder de vista esta relación. Por eso interpreta la soberanía democrática como un rechazo de Dios y como una afirmación de la naturaleza divina del ser humano, es decir, como un proyecto gnóstico. Como afirma Volpi, "la democracia moderna es para Nicolás Gómez Dávila la teología del hombre-dios, ya que ella asume al hombre como Dios y de este principio deriva sus normas, sus instituciones, sus realizaciones" (Volpi 2005, p. 31).

Supongamos por un momento que la hermenéutica de Gómez Dávila es válida: el ser humano se define por su relación con Dios; el individuo soberano es un individuo divinizado. Aun así, la idea de que la soberanía democrática implica divinizar al ser humano podría seguir siendo problemática. La razón de ello se encuentra en la idea misma de soberanía pues, aunque por necesidad es suprema (una soberanía que no sea suprema es una contradicción en los términos), no necesariamente es absoluta: puede no abarcar todos los aspectos de la existencia. Que en una democracia el ser humano sea soberano en materia política no debería llevarnos a pensar que tiene una soberanía absoluta sobre el universo. El humano sólo ocuparía la misma posición de Dios si su soberanía pretendiera abarcarlo todo.

Sin embargo, en los Escolios Gómez Dávila señala que no es sólo en materia política donde podemos encontrar una pretensión soberana: el ser humano moderno aspira a una soberanía ilimitada en todos los

${ }^{3}$ Cfr. con el siguiente texto de Carl Schmitt: "In the theory of the state of the seventeenth century, the monarch is identified with God and has in the state a position exactly analogous to that attributed to God in the Cartesian system of the world" (Schmitt 1985, p. 46).

Revista de Filosofía Diánoia, vol. 65, no. 84 (mayo-octubre de 2020) e-ISSN: 1870-4913 • DOI: https://doi.org/10.22201/iifs.18704913e.2020.84.1612 
aspectos de la existencia; en otras palabras, pretende ser de la misma sustancia que (como se dice en el epígrafe de Heracleón) su plenamente bendita e ingénita naturaleza. Por eso el colombiano señala que "el regnum hominis, con cuya prédica Bacon inaugura el mundo moderno, no es parodia del regnum Dei, sino su versión gnóstica" (Gómez Dávila 2005e, p. 193). En efecto, el regnum hominis ejercería su dominio sobre todo lo que esté a su alcance. No se trata nada más de política, sino de todos los aspectos de la vida humana: ontología, axiología, religión, estética, etc. Ya no hay autoridad por encima del humano: su soberanía es absoluta. Esto quiere decir para el autor que el humano ha ocupado totalmente el papel de Dios. Y no es sólo en la democracia donde se diviniza: la Modernidad misma es un proyecto gnóstico. Esto ya se sugiere en Textos, dado que Gómez Dávila señala que la época democrática es aquella en la que se desarrollan el capitalismo, el comunismo y el Estado soberano, es decir, los aspectos esenciales de la Modernidad. En lenguaje de nuestro filósofo, decir "Modernidad" y "democracia" es decir "gnosticismo". Por lo mismo, el suceso central de la Modernidad, al menos en términos ideológicos, se interpreta también desde la clave gnóstica en los Escolios:

A un dios solo lo encadena la ignorancia. Un dios permanece caído solo mientras ignore ser dios.

Aufklärung es la traducción circunspecta de Gnosis (Gómez Dávila 2005c, p. 193).

Así pues, en los Escolios hay una continuación de la tesis central de Textos: la Ilustración es gnóstica. También vemos que, por lo mismo, hay una conexión entre sus bases hermenéuticas. Por ejemplo, en Escolios dice que:

Desconocer la infraestructura religiosa de toda acción es parecerse hoy a los que ayer negaban su infraestructura económica.

Los marxistas ocupan hoy una posición intelectualmente análoga a la de los antimarxistas de ayer. (Gómez Dávila 2005a, p. 349)

En efecto, aquí se sostiene de nuevo la primacía de la interpretación religiosa para comprender los actos del individuo. Con todo, aunque podamos tener reservas claras frente a la propuesta interpretativa del colombiano, hay que resaltar que su tesis de la Modernidad democrática como una época gnóstica es interesante. Primero, porque no repite la 
vieja tesis de que los conceptos modernos son ideas teológicas secularizadas, sino que es mucho más radical: la Modernidad es una época auténticamente religiosa, aunque lo sea de un modo oculto o insincero. Así, lejos de representar una superación de las ideas teológicas, la Modernidad es una reformulación auténtica de tesis gnósticas. Vista así, nuestra época no es un nuevo comienzo, sino una recaída en el antiguo gnosticismo. Y esto es lo que a Gómez Dávila le inquieta tanto: si el ser humano moderno vive en medio de una inmensa podredumbre espiritual, es precisamente debido a ella. Para un católico preferirse a sí mismo sobre Dios es un pecado gravísimo. De manera paradójica, aquí nuestro autor terminará por interpretar el presente de manera cuasi gnóstica: vivimos en medio de una verdad alienada (la gnóstica Modernidad democrática) de la que debemos escapar para acercarnos al verdadero Dios y así vivir en forma auténtica.

Resulta interesante que el juicio de Gómez Dávila aparezca hoy en día en autores de diversas tendencias ideológicas, lo que indicaría que quizá se puede llegar a la misma conclusión por caminos distintos. Desde la izquierda, Terry Eagleton señala respecto del ateísmo de Nietzsche que éste:

[p]ermanece incompleto. Dado que los significados [...] deben ser hechos por el hombre, es difícil ver cómo esto no vuelve al Übermensch que realiza esta tarea un minidios. Como el todopoderoso, no descansa en nada excepto en sí mismo. No puede haber discusiones sobre la autonomía o la autogeneración sin una mirada a la teología. El hombre sólo puede reemplazar a Dios si él mismo es su autocreador, aboliendo así su dependencia y contingencia; y, sin embargo, el volverse autocreado significa perpetuar la deidad en forma diferente. Es homenajear la religión en un intento de abolirla. (Eagleton 2014, p. 161; la traducción es mía)

Curiosamente, la cita anterior es un resumen exacto de la tesis principal de Gómez Dávila que he expuesto en este artículo. Podría servir de epígrafe en una tesis entera sobre el pensamiento del colombiano. Por otra parte, desde el conservadurismo Rémi Brague afirma algo parecido:

El proyecto moderno aparece como una emancipación de todo lo que se presenta por encima del hombre, como su origen inaccesible: un dios creador y/o legislador, o una naturaleza a la que su carácter activo hace divina. Invierte la percepción del contexto teológico de la antropología. En lugar de ser el hombre el que debe recibir su norma de una autoridad exterior,

Revista de Filosofía Diánoia, vol. 65, no. 84 (mayo-octubre de 2020) e-ISSN: 1870-4913 • DOI: https://doi.org/10.22201/iifs.18704913e.2020.84.1612 
es él el que determina lo que sobre él puede reivindicar una autoridad. La relación entre el hombre y lo divino toma la figura de "él o yo". El humanismo tenderá entonces a realizarse como un ateísmo. (Brague 2015 p. 17)

En todo caso, y más allá de las coincidencias, sería interesante preguntarnos si ese proyecto moderno se ha hecho realidad. Podríamos responder que la soberanía humana no es en realidad tan absoluta como parece en un principio. Aunque Dios ya no actúe sobre el mundo, la Modernidad necesita que alguien o algo aparte del ser humano tome su papel. Esto es algo que el mismo Eagleton ha visto: los sujetos modernos han matado a Dios para entronizarse en "la más espectacular de las revueltas edípicas" (Eagleton 2014 p. 157), pero Dios sigue vivo en las figuras de la Razón, el Hombre, el Progreso y la Moralidad. En otras palabras, seguimos pensando en un Otro que nos da permiso para actuar, que otorga valor a nuestra vida, que decide lo que está bien y mal. La Modernidad ha reemplazado a Dios con otras figuras que ejercen su misma función. ${ }^{4}$ No somos tan autónomos como creemos.

Aquí llegamos a una de las paradojas del mundo moderno: aunque la soberanía humana sea incompatible con el Dios de Gómez Dávila, los modernos siguen creyendo en alguno de sus sustitutos. El materialismo dialéctico, la nación, el capitalismo, el espíritu objetivo, el Ser y otros conceptos han adoptado el papel que antes tenía Dios: funcionan como el gran Otro que da sentido a las vidas de los seres humanos. Así, el asunto es más problemático de lo que había visto nuestro autor: la muerte total de Dios no se ha dado ni, por lo tanto, la divinización completa del humano. Como señala Žižek 2009, la Modernidad no puede funcionar con la muerte de Dios porque ésta implica que nada está permitido. Si nadie ejerce la función de Dios, no hay mundo. Dios, o sus lugartenientes, son necesarios para que tengamos permiso de hacer algo, para que nuestros actos tengan sentido. Paradójicamente, aquí se reafirma la vieja lógica cristiana: el ser humano sólo es autónomo en la medida en que Dios exista. Vista así, la Modernidad no es un proyecto gnóstico: simplemente hemos reemplazado al antiguo Dios con sucedáneos.

${ }^{4}$ A propósito de esta paradoja, recomiendo revisar una de las ideas más interesantes de Latour, a saber, la de que la Modernidad necesita un Dios muerto y un Dios vivo al mismo tiempo (Latour 1991, pp. 33, 34). 


\section{Referencias bibliográficas}

Abad Torres, Alfredo Andrés, 2008, Pensar lo implícito. En torno a Gómez Dávila, Centro de Recursos Informáticos y Educativos, Pereira.

Abad Torres, Alfredo Andrés, 2010, "Nicolás Gómez Dávila y las raíces gnósticas de la Modernidad", Ideas y Valores, vol. 59, no. 142, pp. 131-140, disponible en $<$ https://revistas.unal.edu.co/index.php/idval/article/view/36602 /38523>.

Blumenberg, Hans y Robert M. Wallace, 1983, The Legitimacy of the Modern Age, Mit Press, Cambridge, Mass.

Brague, Rémi, 2015, El reino del hombre. Génesis y fracaso del proyecto moderno, trad. José Antonio Millán Alba, Encuentro, Madrid.

Eagleton, Terry, 2014, Culture and the Death of God, Yale University Press, New Haven.

Eliade, Mircea, 2015, Lo sagrado y lo profano, trad. Luis Gil Fernández y Ramón Alfonso Díez Aragón, Paidós, Barcelona.

Goenaga Olivares, Francia Elena, 2007, "La tumba habitada. Una reflexión sobre 'La Modernidad' en la obra de Nicolás Gómez Dávila", Paradoxa, vol. 7, no. 14 , pp. 85-120.

Gómez Dávila, Nicolás, 2002, Textos I, Villegas, Bogotá.

Gómez Dávila, Nicolás, 2003, Notas, Villegas, Bogotá.

Gómez Dávila, Nicolás, 2005a, Escolios a un texto implícito I, Villegas, Bogotá.

Gómez Dávila, Nicolás, 2005b, Escolios a un texto implícito II, Villegas, Bogotá.

Gómez Dávila, Nicolás, 2005c, Nuevos escolios a un texto implícito I, Villegas, Bogotá.

Gómez Dávila, Nicolás, 2005d, Nuevos escolios a un texto implícito II, Villegas, Bogotá.

Gómez Dávila, Nicolás, 2005e, Sucesivos escolios a un texto implícito I, Villegas, Bogotá.

Latour, Bruno, 1991, We Have Never Been Modern, trad. Catherine Porter, Harvard University, Cambridge.

Marx, Karl, 2019, Diferencia de la filosofía de la naturaleza en Demócrito y Epicuro, Ayuso, Madrid.

Molina, Tomás, 2019a, "Montaigne y Burckhardt como fuentes de la doctrina reaccionaria en los Escolios de Nicolás Gómez Dávila", Revista Escritos, vol. 27, no. 58, pp. 1-21, <https://doi.org/10.18566/escr.v27n58.a03>.

Molina, Tomás, 2019b, "Los epígrafes en los Escolios de Nicolás Gómez Dávila: hacia una lectura intertextual", Universitas Philosophica, vol. 73, año 36, pp. 237-256, <https://doi.org/10.11144/Javeriana.uph36-73.engd $>$.

Mosebach, Martin, 2017, "Un ermitaño en las orillas del mundo habitado. Sobre Nicolás Gómez Dávila", trad. Tomás Molina, Revista Nova et Vetera, vol. 3, no. 29.

Onfray, Michel, 2007, Los ultras de las luces. Contrahistoria de la filosofía 4, trad. Marco Aurelio Galmarini, Anagrama, Barcelona. 
Philpott, Daniel, 2003, "Sovereignty", en Edward N. Zalta (coord.), The Stanford Encyclopedia of Philosophy, disponible en $<$ https://plato.stanford.edu/ entries/sovereignty/>.

Pizano, Francisco, 1991, "Don Nicolás Gómez Dávila”, El Tiempo.

Rabier, Michael, 2014a, "La 'cuestión literaria' en la obra de Nicolás Gómez Dávila”, Perífrasis. Revista de Literatura, Teoría y Crítica, vol. 5, no. 10, pp. 25-40.

Rabier, Michael, 2014b, "Nicolás Gómez Dávila y las paradojas del conservadurismo", La Razón Histórica. Revista Hispanoamericana de Historia de las Ideas Políticas y Sociales, vol. 26, pp. 226-243.

Rabier, Michael, 2016, Philosophie, gnose et modernité. Nicolás Gómez Dávila lecteur d'Éric Voegelin, tesis de doctorado, Université Paris-Est, París.

Schmitt, Carl, 1985, Political Theology: Four Chapters on the Concept of Sovereignty, trad. George Schwab, University of Chicago Press, Chicago.

Serrano Marín, Vicente (trad.), 1995, "Carta de Jacobi a Fichte sobre el nihilismo", Anales del Seminario de Historia de la Filosofía, vol. 12, pp. 235-263.

Serrano, José Miguel, 2015, Democracia y nihilismo. Vida y obra de Nicolás Gómez Dávila, Universidad de Navarra, Pamplona.

Severino, Emanuele, 2001, "L'universo visto in sogno", Corriere della Sera, 6 de mayo, p. 31.

Torres Duque, Óscar, 1995, "Nicolás Gómez Dávila: la pasión del anacronismo", Boletín Cultural y Bibliográfico, vol. 32, no. 40, pp. 31-49, disponible en $<$ https://publicaciones.banrepcultural.org/index.php/boletin_cultural/artic le/view/1858>.

Volpi, Franco, 2005, "Nicolás Gómez Dávila. El solitario de Dios”, en Nicolás Gómez Dávila, Escolios a un texto implícito I, Villegas, Bogotá.

Žižek, Slavoj, 2009, "How to Read Lacan", en Lacan dot com, disponible en $<$ http://www.lacan.com/essays/?p=82>.

Recibido el 15 de febrero de 2019; revisado el 25 de julio de 2019; aceptado el 22 de agosto de 2019. 